\title{
Clonotypic Heterogeneity of Lewis Rat T Cells Specific for the Encephalitogenic 68-86 Region of Myelin Basic Protein ${ }^{1}$
}

\author{
M. D. Mannie, ${ }^{* 2}$ P. Y. Paterson, $\dagger^{, 3}$ D. C. U'Prichard $¥$ and D. W. Thomas* \\ *Department of Microbiology and Immunology, University of Michigan Medical School, Ann Arbor, \\ Michigan 48109; $\uparrow$ Department of Microbiology-Immunology, Medical and Dental Schools, Northwestern \\ University, Chicago, Illinois 60611 and Department of Neurobiology and Physiology. College of Arts and \\ Sciences, Northwestern University, Evanston, Illinois 60201; and $\ddagger I C I$ Pharmaceuticals Group, \\ ICI Americas Inc., Wilmington, Delaware 19897
}

Received April 5, 1989; May 31, 1989

Experimental autoimmune encephalomyelitis was induced in a Lewis rat by sensitization with synthetic peptide GP68-86, representing the 68-86 sequence of guinea pig myelin basic protein (GPMBP). To delineate T cell determinants of GP68-86, lymph node cells from this rat were activated in culture with GP68-86 and were fused with cells of the mouse thymoma BW5147. The resultant hybrids were cloned by limiting dilution and screened for GP68-86evoked secretion of IL 2 in the presence of rat splenocytes. Twelve T cell hybrids derived in this manner were tested for reactivity to different heterologous species of MBP as well as to substituted or truncated analogs of GP68-86. The hybrids generally exhibited potent reactivity to GPMBP but differed markedly in their reactivity to autologous rat MBP (RMBP). A few exceptional hybrids exhibited crossreactivity with peptides in which native serine ${ }^{75}$ or serine ${ }^{80}$ residues of GPMBP were substituted with either alanine ${ }^{75}\left(\mathrm{~A}^{75}\right)$ or proline $\mathrm{e}^{80}\left(\mathrm{P}^{80}\right)$ residues. These crossreactive hybrids also possessed high levels of anti-RMBP reactivity. The remaining hybrids were unresponsive to the $\mathrm{A}^{75}$ and $\mathrm{P}^{80}$ substituted peptides and, with one exception, had relatively low levels of anti-RMBP reactivity. Unique reactivity patterns were also revealed by hybrid responses to peptides having modified C-terminal 84-86 residues. In summary, the contrasting fine specificities of different hybrids indicated that several distinct clones of $T$ cells mediate the immune response of Lewis rats against the 68-86 region of GPMBP. Furthermore, heterogeneity in the hybrid response to "self" RMBP may reflect substantial differences in encephalitogenic potency of the T cell clones from which these hybrids were derived. @ 1989 Academic Press, Inc.

\section{INTRODUCTION}

Experimental autoimmune encephalomyelitis (EAE) ${ }^{4}$ in Lewis rats is mediated by $T$ lymphocytes specific for encephalitogenic determinants in the 68-86 region of

\footnotetext{
' This investigation was supported by NIH Grants AI-19273 and NS 06262, by a Postdoctoral Fellowship from the National Multiple Sclerosis Society (Grant FG 758-A-1), and by The Mulvihill Family Foundation in Memory of Rosemary Mulvihill Speth.

${ }^{2}$ Dr. Mark D. Mannie is a Postdoctoral Fellow of the National Multiple Sclerosis Society.

${ }^{3}$ Dr. Philip Y. Paterson is a Javits Neuroscience Investigator.

${ }^{4}$ Abbreviations used: B, bovine; EAE, experimental autoimmune encephalomyelitis; GP, guinea pig; LAS, LSP, and LSS, Lewis rat hybrids responsive to $\left[\mathrm{A}^{75}, \mathrm{~S}^{80}\right] \mathrm{GP} 68-84,\left[\mathrm{~S}^{75}, \mathrm{P}^{80}\right] \mathrm{GP68-84}$, and $\left[\mathrm{S}^{75}, \mathrm{~S}^{80}\right]-$ GP68-84, respectively; LNC, lymph node cells; MB, modified bovine; MBP, myelin basic protein; and R, rat.
} 
MBP (1-6). The disease-inducing activity of this region can be profoundly affected by subtle structural changes in primary structure (7). For example, the $68-86$ sequence of guinea pig myelin basic protein (GPMBP) is substantially more encephalitogenic in rats than the corresponding sequence of rat MBP (RMBP) (1). These two sequences differ by only a single methyl group in that the former contains a $S^{80}$ residue $^{5}$ whereas the latter contains a $T^{80}$ residue. As with encephalitogenic activity, the 68-88 sequence of GPMBP is substantially more active than the corresponding RMBP sequence for the stimulation of MBP-specific in vitro proliferation (1).

In previously reported experiments designed to further characterize antigen specificity of encephalitogenic T cells, Lewis rat lymph node cells (LNC) were sensitized and then tested for in vitro responsiveness to different substituted analogs of GP68$84(6,8)$. The specificity of encephalitogenic LNC was determined by testing whether these LNC became activated in culture with MBP-related antigens, as measured by their ability to potently transfer EAE to naive recipients. Unexpectedly, the specificity pattern observed for encephalitogenic responses differed substantially from the pattern observed for in vitro proliferation. Encephalitogenic determinants that potentiated EAE transfer activity of sensitized LNC were permissive of single residue substitutions in which native GPMBP residues $S^{75}$ and $S^{80}$ were replaced with either $A^{75}$ or $\mathrm{P}^{80}$ residues. In contrast, determinants stimulating in vitro proliferation of sensitized LNC were completely disrupted by the same substitutions. These results indicated that two functionally distinct $\mathrm{T}$ cell subsets, as defined by their reciprocal abilities to mediate either EAE transfer or proliferation, exhibited distinct clonotypic specificities for GP68-86-related peptides.

In the present investigation, the clonotypic heterogeneity of the Lewis rat $\mathrm{T}$ cell response against GP68-86 was studied to determine how previously described $T$ cell subset specificities $(6,8)$ correlate with the fine specificity of individual T lymphocyte clones. For this purpose, monoclonal T cell hybrids specifically reactive with GP6886 were derived to delineate $T$ cell determinants of the encephalitogenic region. A proportion of these hybrids would predictably recognize major EAE-associated determinants of MBP, since GP68-86 possessed disease-inducing activity approximating that of intact GPMBP (manuscript submitted). The specificity of each hybrid was defined by testing for reactivity to different species of MBP and a set of peptides incorporating sequence deletions and substitutions in GP68-86.

The hybrids were found to express several markedly different specificities. A small number of hybrids expressed specificities which were strikingly similar to specificities previously associated with the potentiation of EAE transfer activity (8). These hybrids exhibited strong reactivity to peptides containing single substitutions of either $\mathrm{A}^{75}$ or $\mathrm{P}^{80}$ residues. These hybrids also possessed potent reactivity to RMBP. In contrast, the majority of hybrids expressed a specificity very similar to that previously associated with in vitro proliferation (8). That is, these hybrids were characterized by a total lack of reactivity with $\mathrm{A}^{75}$ or $\mathrm{P}^{80}$ substituted peptides. Furthermore, these hybrids generally exhibited less cross-reactivity with RMBP. Taken together, these rcsults demonstrate that the immune response associated with the induction of EAE in Lewis rats involves recognition of overlapping determinants in GP68-86 by clonally diverse T cells. In addition, the clonotypically unique specificities of $T$ cell hybrids were found

\footnotetext{
${ }^{5}$ Standard single letter codes designate amino acid residues.
} 
TABLE 1

Heterologous MBPs and MBP-Related Synthetic Peptides

\begin{tabular}{|c|c|c|c|}
\hline \multirow[t]{2}{*}{ Designation } & \multicolumn{2}{|c|}{ Primary structure $^{a}$} & Lot No. ${ }^{b}$ \\
\hline & 7 & 8 & \\
\hline & 2 & 46 & \\
\hline GPMBP & $-Y G S L P Q K S Q\{$ & \}$R$ S Q D E N P V - & \\
\hline RMBP & $---------i$ & \}$-T-\cdots--\cdots$ & \\
\hline Bovine MBP & $-------\mathrm{A}-\mathrm{G}$ & $\mathrm{H}-\mathrm{P}----\cdots$ & \\
\hline Rabbit MBP & $-\cdots-----\{\} \mathrm{G}$ & $\mathrm{H}-\mathrm{P}-----\cdots$ & \\
\hline GP68-86 & $-------\frac{1}{2}$ & \}------- & UCB-R393B \\
\hline$\left[G^{84}\right] G P 68-86$ & $---\cdots-\cdots-1$ & \}$----G--$ & UCB-R381 \\
\hline GP72-86 & ----\{ & \}$-\cdots-\cdots-\cdots$ & UCB-R393A \\
\hline GP75-86 & -1 & \}$-----\cdots$ & PL-005709 \\
\hline GP75-84 & --\{ & \}$--\cdots-\cdots$ & PL-005063 \\
\hline GP68-84 & $----\cdots---\{$ & \}------ & PL-005757 \\
\hline$\left[\mathrm{P}^{80}\right]$ GP68-84 & $--\cdots-\cdots-\cdots$ & $\xi-P---$ & PL-007173 \\
\hline$\left[\mathrm{A}^{75}\right] \mathrm{GP} 68-84$ & $-----A-\{$ & \}$--\cdots--$ & PL-007407 \\
\hline MB68-84 & $-----\cdots$ & \}$-P----$ & PL-004952 \\
\hline MB68-84G & $------A-\{$ & \}$-P---G$ & PL-006142 \\
\hline
\end{tabular}

${ }^{a}$ Dashes represent identity with amino acid sequence of GPMBP. Deletions have been denoted as empty spaces enclosed by brackets \{\} . The numbering of residue positions follows the original system introduced by Eylar et al. for bovine MBP (10).

${ }^{b}$ Designations "UCB" or "PL" indicate that respective peptides were purchased from either UCB Bioproducts S.A. or Peninsula Laboratories.

to correlate closely with LNC specificities previously associated with either potentiation of EAE transfer activity or in vitro proliferation (8).

\section{MATERIALS AND METHODS}

Rats. Lewis rats were purchased from Harlan Sprague-Dawley, were maintained in our breeding facility, and were 6-10 weeks of age when used for experiments.

Antigens. MBP was purified from rat or guinea pig spinal cords by the method of Swanborg et al. (9). Bovine and rabbit MBP were kindly provided by Dr. Robert Zand (University of Michigan). In addition, bovine and rabbit MBP were purchased from Sigma and used in some experiments. The peptides were custom synthesized and purified by either Peninsula Laboratories (Belmont, CA) or UCB Bioproducts S. A. (Belgium). The homogeneity of each peptide was verified by analytical HPLC (6). The designations and primary structure of the MBPs and synthetic peptides used in this investigation are presented in Table 1.

Sensitization of a Lewis rat with GP68-86. A Lewis rat was sensitized with $0.1 \mathrm{ml}$ of an emulsion containing $10 \mathrm{nmol}$ of GP68-86 and $200 \mu \mathrm{g}$ Mycobacterium tuberculosis H37Rv, Jamaican strain, in complete Freund's adjuvant. Fifty microliters of the sensitizing inoculum was injected intradermally into each hindleg footpad.

In vitro proliferation of GP68-86-sensitized LNC. GP68-86-sensitized LNC were cultured in 96-well microtiter plates at a cell density of $5 \times 10^{5}$ cells/well $(167 \mu \mathrm{l})$ in RPMI 1640 medium supplemented with 10\% FBS (GIBCO), $2 \mathrm{~m} M$ glutamine (Sigma), $100 \mu \mathrm{g} / \mathrm{ml}$ streptomycin (GIBCO), $100 \mathrm{units} / \mathrm{ml}$ penicillin (GIBCO), and 50 
$\mu M$ 2-mercaptoethanol (Eastman-Kodak) at $37^{\circ} \mathrm{C}$ and $5 \% \mathrm{CO}_{2}$. After 72 hours of culture, each well was pulsed with $1 \mu \mathrm{Ci}(20 \mu \mathrm{l})$ of $\left[{ }^{3} \mathrm{H}\right]$ thymidine $(6.7 \mathrm{Ci} / \mathrm{mmol}$; NEN). The cultures were harvested after an additional $24 \mathrm{hr}$ of culture. Incorporation of $\left[{ }^{3} \mathrm{H}\right]$ thymidine was measured by scintillation counting.

Generation of Thybrids specifically reactive with GP68-86. Rat-mouse T cell hybrids were derived according to modifications of the method described by Prud'homme et al. (11). Draining LNC were excised from one donor rat 14 days after sensitization with GP68-86 in complete Freund's adjuvant, while the rat was exhibiting severe clinical signs of acute EAE. The LNC were cultured with $0.5 \mu M$ GP68-86 for 2 days and then washed extensively in Hanks' buffered saline solution. LNC (40 $\times 10^{6}$ ) were fused with $20 \times 10^{6} \mathrm{BW} 5147$ thymoma cells by mixing in the presence of $50 \%$ PEG (v/v) for $2 \mathrm{~min}$. The cells were washed and resuspended in selective HAT medium (complete RPMI 1640 supplemented with $60 \mu M$ hypoxanthine, $30 \mu M$ thymidine, and $400 \mathrm{nM}$ aminopterin) that was supplemented with IL 2-containing supernatants from Concanavalin A (Miles-Yeda)-stimulated Lewis rat splenocytes. The hybrids were cloned by limiting dilution in 96 -well plates. Growth-positive wells were screened for GP68-86-evoked IL 2 production as described below. Hybrids exhibiting responsiveness to GP68-86 were cultivated in complete RPMI 1640 medium in the absence of exogenous IL 2. Each hybrid was then subcloned by limiting dilution in 96-well plates.

Antigen-evoked IL 2 production by $T$ cell hybridomas. $T$ cell hybrids were aliquoted into 96-well plates at a cell density of $10^{5}$ cells/well and were cultured with $5 \times 10^{5}$ syngeneic splenocytes and antigen. After $24 \mathrm{hr}, 100 \mu \mathrm{l}$ of culture supernatant was transferred to new wells containing cells of the IL 2-dependent CTLL indicator line. These cells were pulsed with $1 \mu \mathrm{Ci}$ of $\left[{ }^{3} \mathrm{H}\right]$ thymidine after $24 \mathrm{hr}$ in culture and then harvested on fiberglass filters after an additional $24 \mathrm{hr}$. $\left[{ }^{3} \mathrm{H}\right]$ Thymidine incorporation in DNA was quantitated by liquid scintillation counting.

Rationale and experimental approach. This investigation focused on whether monoclonal $\mathrm{T}$ cell specificities correlate with either of the specificity patterns previously observed for encephalitogenic and proliferative subsets of $\operatorname{LNC}(6,8)$. As part of this analysis, the proliferative responses of GP68-86-sensitized LNC were assessed side by side with those for monoclonal $\mathrm{T}$ cell hybrids. The specificities of GP68-86sensitized LNC and T cell hybrids were defined by their reactivity to MBPs and peptides that differed in either concise deletions of the $\mathrm{N}$ - or $\mathrm{C}$-termini or single substitutions of internally located residues (see Table 1). With the range of peptides and MBPs studied, information was obtained about all residues in the 68-86 region, except positions 76,79 , and 81-83. If a given alteration in sequence affected a $T$ cell response, then we concluded that residues therein were either directly recognized or conformationally associated with the respective determinant.

\section{RESULTS}

In vitro proliferation and EAE transfer activity of GP68-86-sensitized $L N C$. As illustrated in Fig. 1, GP68-86-sensitized LNC exhibited potent reactivity with GPMBP and GP68-86. However, the same LNC expressed intermediate reactivity against RMBP and GP68-84 but little or no reactivity against $\left[\mathrm{A}^{75}\right] \mathrm{GP} 68-84$ and $\left[\mathrm{P}^{80}\right] \mathrm{GP} 68$ 84. After culture with GP68-86 or GP68-84, the adoptive transfer of $30 \times 10^{6} \mathrm{LNC}$ induced paralytic signs of EAE in each of three recipients. Parallel cultures of GP68- 


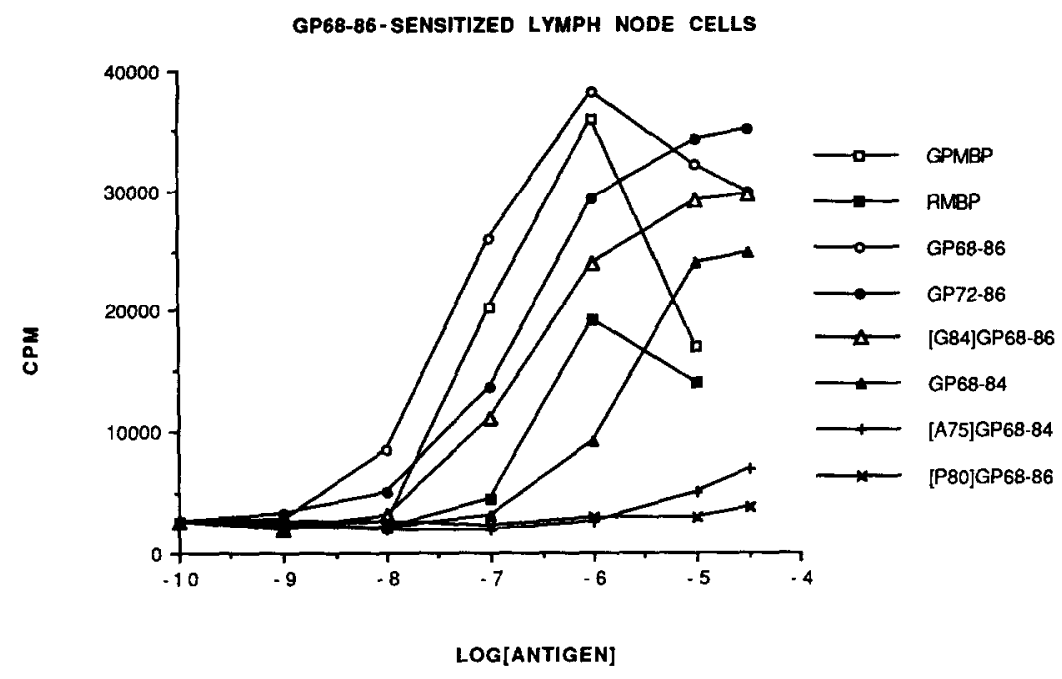

FIG. 1. Proliferative response of GP68-86-sensitized LNC. LNC from draining lymphatics of rats sensitized to GP68-86 were cultured with the designated antigens for 4 days and pulsed with $1 \mu \mathrm{Ci}\left[{ }^{3} \mathrm{H}\right]$ thymidine during the last $24 \mathrm{hr}$. LNC were harvested by filtration and incorporation of $\left[{ }^{3} \mathrm{H}\right]$ thymidine was measured by scintillation counting.

86-sensitized LNC which were not exposed to an activating antigen did not induce EAE when $40 \times 10^{6}$ cells were transferred into two recipients each.

Derivation and specificity of $T$ hybrid cells. Of the 82 rat-mouse $T$ cell hybrids tested, 21 hybrids (25.6\%) secreted IL 2 when cultured with GP68-86 and Lewis rat splenocytes. Another 9 hybrids (11\%) secreted IL 2 when cultured with Lewis rat splenocytes in the absence of any exogenously added antigen.

Twelve GP68-86-specific hybrids exhibited stable growth and reactivity to GP6886 and were thereby chosen for further study. These hybrids exhibited several unique specificity patterns and were labeled as follows: (a) $L A S-A$, Lewis rat $\mathrm{T}$ cell hybrid reactive to $\left[\mathrm{A}^{75}, \mathrm{~S}^{80}\right] G P 68-84$; (b) $L S P$ - $A$, reactive to $\left[\mathrm{S}^{75}, \mathrm{P}^{80}\right] G P 68-84$; (c) $L S S-A$, $L S S-B, L S S-C, L S S-D$, reactivity restricted to native $\left[S^{75}, S^{80}\right] \mathrm{GP} 68-84$ with decreasing levels of reactivity to $R M B P$, respectively.

The LAS determinant. The LAS-A 1 hybrid was the only hybrid recognizing a determinant not disrupted by the $A^{75}$ substitution in $\left[A^{75}\right]$ GP68-84 (Fig. 2). Furthermore, LAS-A 1 potently reacted with RMBP, indicating that the $T^{80}$ residue of RMBP was not critical for this determinant. Of the other structural modifications investigated, only the $\mathrm{P}^{80}$ substitution (Fig. 2) and the 72-74 sequence deletion (Table 2) adversely affected integrity of the LAS determinant. Compared to the other hybrids, LAS-A1 recognized one of the most concise and centrally located determinants in GP68-86.

The LSP determinant. The unique feature of the LSP specificity was the positive response stimulated by $\left[\mathrm{P}^{80}\right] \mathrm{GP} 68-84$. This specificity was represented by two hybrids (Fig. 2). Like LAS-A1, LSP hybrids also exhibited potent reactivity to RMBP. Thus, LSP recognized the only determinant not disrupted by either of the single-residue substitutions at position $80\left(\mathrm{~T}^{80} / \mathrm{P}^{80}\right.$ for $\left.\mathrm{S}^{80}\right)$. The two structural alterations disrupting this determinant were the $A^{75}$ substitution (Fig. 2) and the 72-74 sequence deletion (Table 2). 

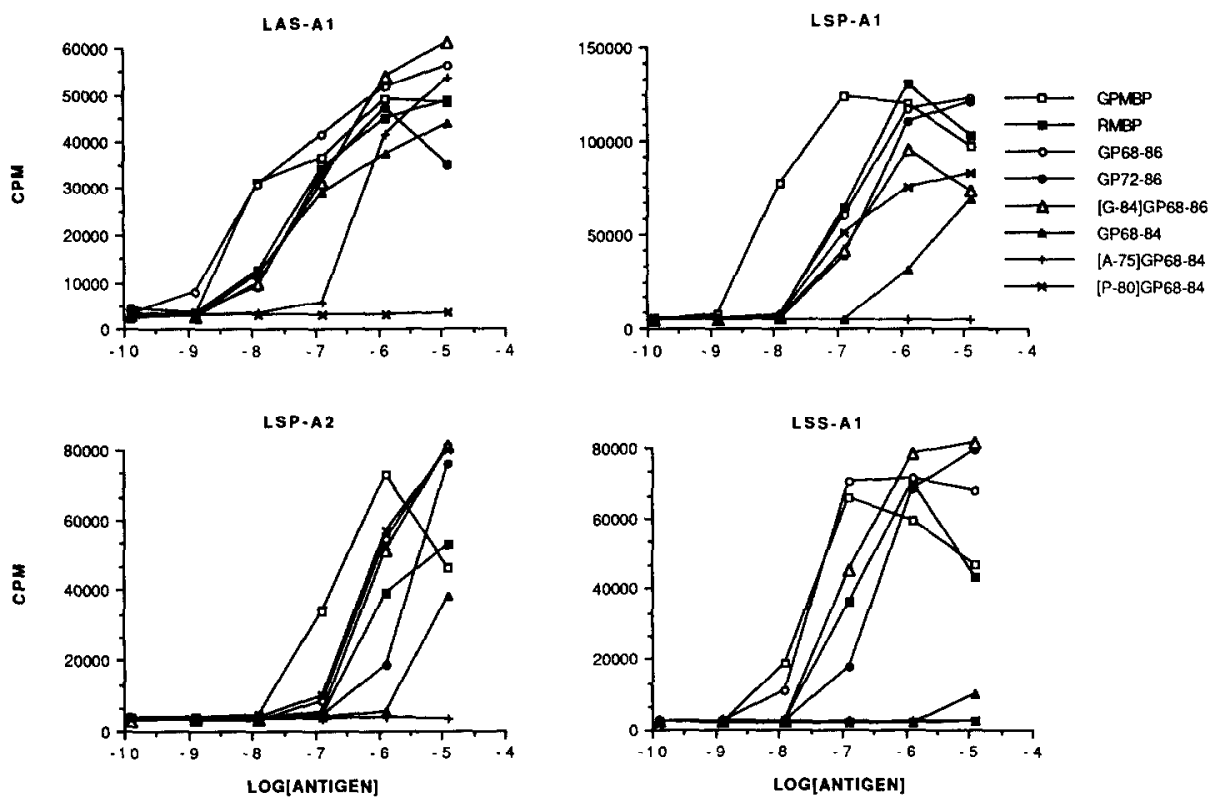

FIG. 2. The LAS, LSP, and LSS-A hybrid specificities. Hybrids were cultured with Lewis rat splenocytes for $24 \mathrm{hr}$ in the presence of the designated antigens. Supernatants from hybrid cultures were tested for IL 2 activity with the CTLL indicator cell line. This line was not sensitive to IL 4 (data not shown). The LASA1, LSP-A1, LSP-A2, and LSS-A1 responses are representative of four, seven, one, and five experiments.

The C-terminal region was implicated as part of the LSP determinant by virtue of significant potency differences distinguishing GP68-86 from GP68-84 and GPMBP from GP68-86 (Fig. 2). In all likelihood, the N-terminal region did not account for differences between GPMBP and GP68-86, since LSP hybrids did not distinguish GP68-86 from GP72-86. The $\mathrm{P}^{80}$ residue, when substituted into GP68-84, conferred an augmentation in activity similar in extent to that conferred by the addition of the C-terminal 85-86 residues. In other words, $\left[\mathrm{P}^{80}\right]$ GP68-84 and GP68-86 were equipotent and both were substantially more active than GP68-84.

The LSS set of determinants. As described in more detail below, the LSS hybrids recognized a diverse set of determinants which were completely disrupted by the substitutions in $\left[\mathrm{A}^{75}\right]$ GP68-84 and $\left[\mathrm{P}^{80}\right]$ GP68-84 (Figs. 2-5). The LSS determinants were recognized by 9 of the 12 hybrids and were also completely disrupted by the 72-74 sequence deletion (Table 2). These hybrids were divided into four categories according to their unique specificities for different MBP-related antigens. Most LSS hybrids (8/9 hybrids) recognized closely related determinants of GPMBP that were significantly impaired by the naturally occurring substitution of $T^{80}$ for $S^{80}$ in RMBP.

The LSS-A determinant. The LSS-A1 hybrid was the only LSS hybrid exhibiting highly potent responses to RMBP (Fig. 2). Thus, like the LAS and LSP determinants, the LSS-A determinant was potentially a very active autoantigen. Another unique feature of the LSS-A1 determinant was the abruptly different requirements for the $\mathrm{N}^{84}$ side chain as compared to the 85-86 sequence. The $\mathrm{N}^{84}$ side chain deletion of $\left[\mathrm{G}^{84}\right] \mathrm{GP} 68-86$ was without significant consequence whereas the truncation of the adjoining PV 85-86 sequence was severely disruptive. 
TABLE 2

General Features of Determinants Recognized by T Cell Hybrids

Stimulation indices ${ }^{a}$ of GP68-86-sensitized LNC or CTLLs cultured with supernatants from $T$ cell hybrids

\begin{tabular}{lrrrrrrr}
\cline { 2 - 7 } Antigen $^{b}$ & LNC $^{c}$ & LAS-A1 $^{d}$ & LSP-A1 & LSS-A1 & LSS-B2 & LSS-C2 & LSS-D2 \\
\hline GPMBP & 15.7 & 42.0 & 82.5 & 95.4 & 122.3 & 169.0 & 109.1 \\
Bovine MBP & ND & 1.2 & 0.9 & 1.0 & 1.4 & 1.5 & 1.5 \\
Rabbit MBP & ND & 1.0 & 1.5 & 1.0 & 1.4 & 2.5 & 1.7 \\
MB68-84 & 1.4 & 1.8 & 1.3 & 1.7 & 1.2 & 1.9 & 1.3 \\
MB68-84G & 1.6 & 1.5 & 2.0 & 0.7 & 1.9 & 1.5 & 1.1 \\
GP75-86 & 1.4 & 1.1 & 1.0 & 0.7 & 1.0 & 2.2 & 0.7 \\
GP75-84 & 1.3 & ND & 1.6 & 0.4 & 1.9 & 1.0 & 0.5 \\
\hline
\end{tabular}

a Stimulation index was defined as the ratio of the mean cpm of quadruplet wells with antigen to mean cpm of quadruplet welis without antigen.

${ }^{b}$ Antigens were added to culture at 1 and $10 \mu M$ concentrations. The antigen concentration stimulating the greatest degree of proliferation was listed in the table above.

${ }^{c}$ GP68-86-sensitized LNC were cultured for 3 days in the presence of the designated antigens and were then pulsed with $1 \mu \mathrm{Ci}\left[{ }^{3} \mathrm{H}\right.$ ]thymidine for an additional day. The $\mathrm{LNC}$ were harvested and $\left[{ }^{3} \mathrm{H}\right.$ ] thymidine incorporation was measured by scintillation counting.

${ }^{d}$ The $\mathrm{T}$ hybrids were stimulated with the designated antigens in the presence of Lewis rat splenocytes for $24 \mathrm{hr}$. Supernatants from these hybrid cultures were tested for IL 2 content by measuring [ ${ }^{3} \mathrm{H}$ ] thymidine incorporation in cell cultures of IL 2-dependent CTLLs.

The LSS-B determinant. Much like LSS-A1, the LSS-B hybrids exhibited potent responses to GPMBP, GP68-86, GP72-86, and $\left[\mathrm{G}^{84}\right]$ GP68-86. The responses of the three LSS-B hybrids are illustrated in Fig. 3. However, unlike LSS-A1, the LSS-B hybrids exhibited responses to GP68-84 and RMBP that were 10-100 times less potent than the respective responses to GP68-86 and GPMBP. Thus, these determinants differed markedly in their requirement for the $\mathrm{S}^{80}$ residue of GPMBP and the PV 8586 sequence of GP68-86. Also, LSS-B hybrids, in all experiments (6/6), exhibited responses to GP72-86 that were slightly more potent than responses against GP6886 . This is in contrast to the other hybrids, where in all experiments (16/16) GP6886 was more potent than GP72-86.

The LSS-B3 hybrid exhibited a pattern of reactivity that was similar to but rightshifted relative to that of LSS-B1 and LSS-B2. In fact, LSS-B3 appeared to be generally less reactive to GPMBP than any of the other hybrids characterized in this study. For example, LSS-B3 reliably exhibited half-maximal responses when cultured with approximately $320 \mathrm{n} M$ GPMBP whereas the other hybrids generally required only 3.2-32 $\mathrm{n} M$ GPMBP for half-maximal responses. Even though LSS-B3 was less responsive to GPMBP, when stimulated by optimal concentrations of antigen, the hybrid secreted IL 2 in amounts similar to that secreted by the other hybrids. Possibly, LSS-B3 lacked functional accessory molecules such as CD2 or CD4 necessary for high-affinity interactions with antigen-presenting cells.

The LSS-C determinant. The LSS-C determinant was recognized by 2 of 12 hybrids and was adversely affected by many of the structural alterations studied (Fig. 4). Deletion of the C-terminal 85-86 sequence or the $T^{80}$ substitution in RMBP resulted in a 100 - to 1000 -fold reduction in potency. The $\mathrm{G}^{84}$ substitution in $\left[\mathrm{G}^{84}\right] \mathrm{GP} 68-86$ consis- 

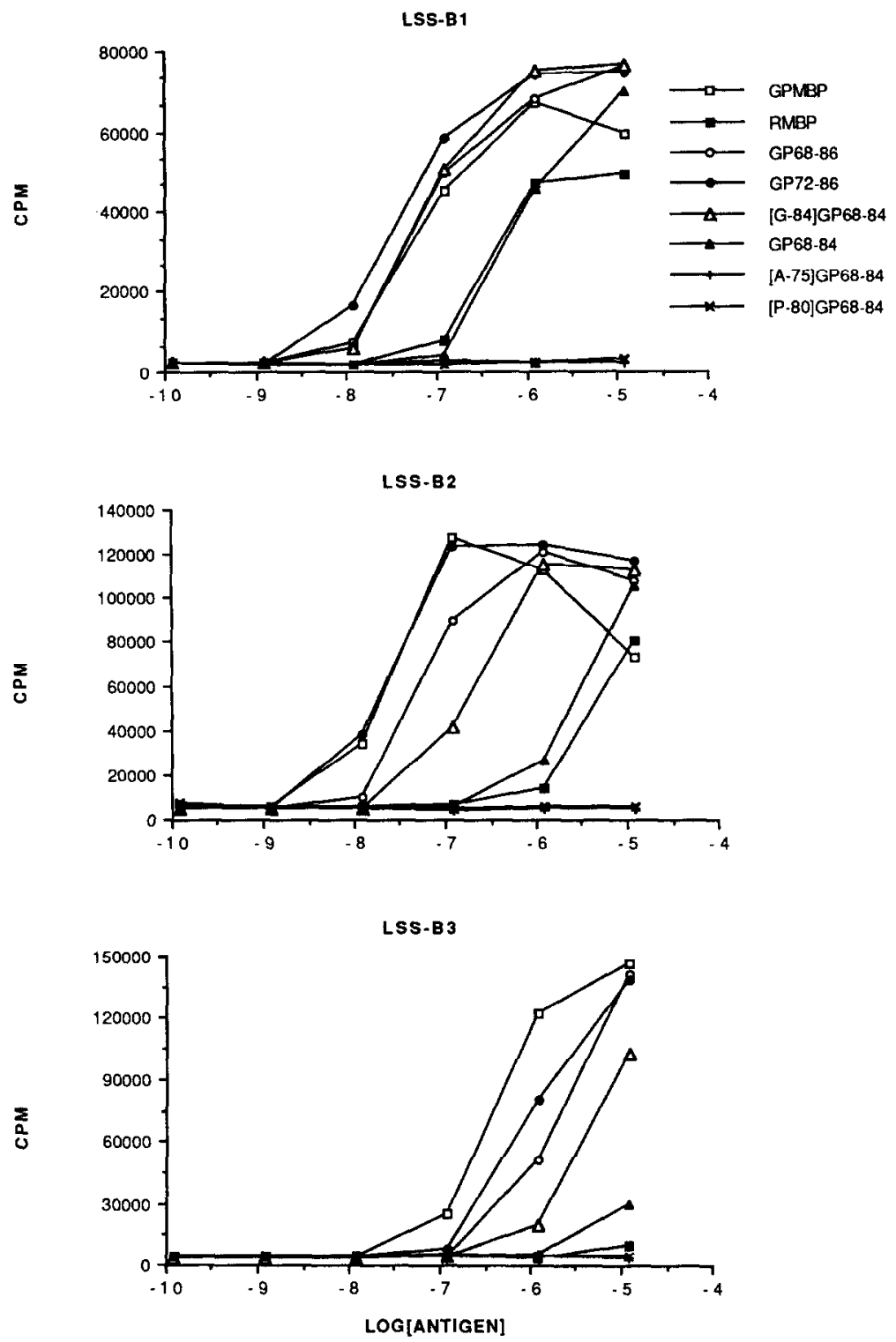

FIG. 3. The LSS-B hybrid specificities. The LSS-B1, B2, and B3 specificities are representative of a total of five, one, and six experiments, respectively.

tently resulted in an approximate 10 -fold reduction in potency. Thus, unlike the previously described determinants, the LSS-C determinant appeared continuous, comprising a linear array of contiguous amino acid residues.

The LSS-D determinant. This determinant was recognized by 3 of 12 hybrids (Fig. 5) and probably possessed the least encephalitogenic potential in that the LSS-D response to RMBP was approximately 1000 times less than GPMBP. The C-terminal portion of the 68-86 sequence appeared to be especially important in the formation of this determinant. For the LSS-D hybrids, $\left[G^{84}\right]$ GP68-86 was 10 - to 100 -fold less 


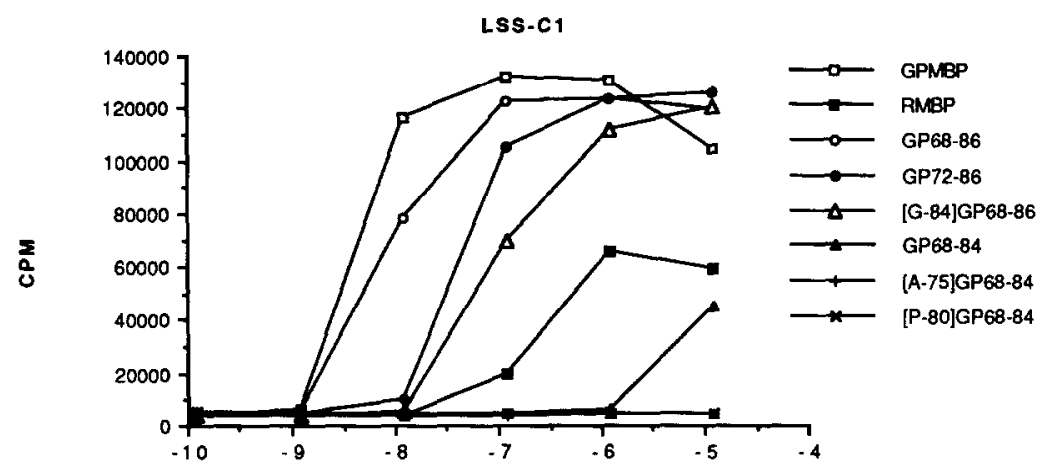

LSS.c2

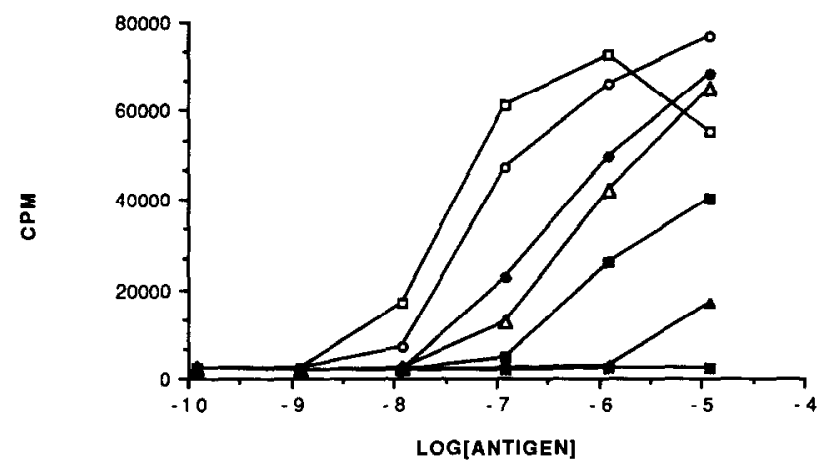

FIG. 4. The LSS-C hybrid specificities. The LSS-C1 and C2 specificities are representative of a total of three and four experiments, respectively.

active than GP68-86, and GP68-84 did not stimulate any response. The possibility that LSS-D determinants included C-terminal residues beyond position 86 was suggested by the large activity differences among GPMBP, GP68-86, and GP68-84. The relatively small activity difference between GP68-86 and GP72-86 indicated that the N-terminus was not vital in the formation of the LSS-D determinant. Overall, this determinant appeared least able to accommodate alterations in the native GPMBP sequence while also having the most diffuse format, extending the length of GP7286 and quite probably beyond.

General features of $T$ cell determinants in GP68-86. Several structural modifications of GP68-86, including the deletion of the 72-74 sequence or insertion of the bovine MBP 76-77 G-H sequence, severely impaired recognition of this region by all of the hybrids used in this study (Table 2). These results indicate that the encephalitogenic region of MBP is a cluster of overlapping $T$ cell determinants which invariably include residues in the 72-74 and 75-80 MBP sequences.

In other studies, encephalitogenic Lewis rat $\mathrm{T}$ cells have been shown to recognize MB68-84 and MB68-84G (6). In addition, Lewis rat $T$ cell hybrids have exhibited responses to the 69-87 region of rabbit MBP (12). These additional clonotypes, although not observed in this investigation, would indicate that yet further diversity characterizes the Lewis rat $\mathrm{T}$ cell response to the encephalitogenic region of MBP. 

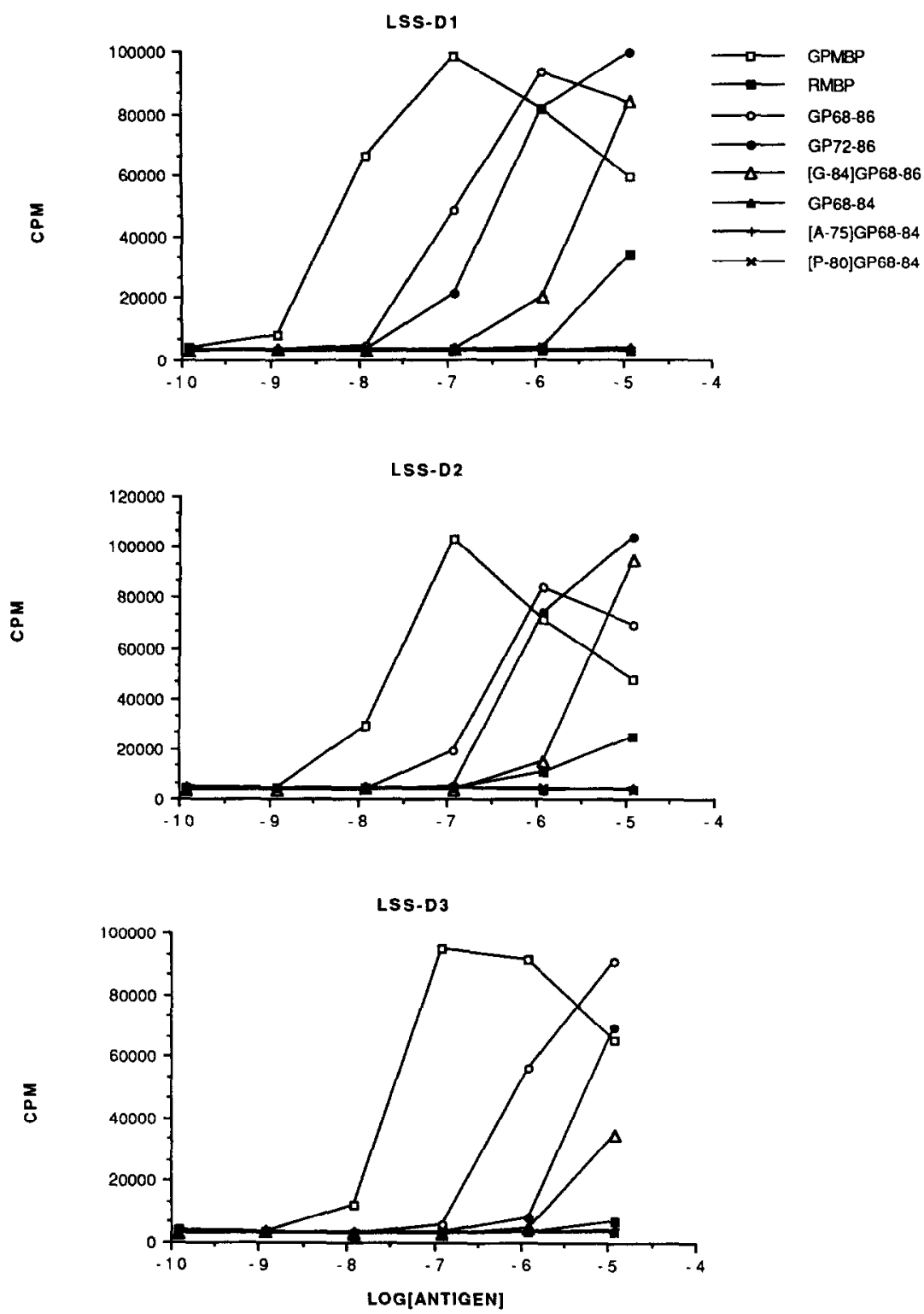

FIG. 5. The LSS-D hybrid specificity. The LSS-D1, D2, and D3 specificities are representative of a total of four, five, and six experiments, respectively.

\section{DISCUSSION}

The finding that six discrete categories of specificity comprised the anti-GP68-86 hybrid response provided several novel insights into the antigenic structure of the encephalitogenic region. First, Lewis rat $T$ cells specifically recognize multiple overlapping $T$ cell determinants in the encephalitogenic region of GPMBP (Figs. 2-5; also see summary diagram in Fig. 6). Second, T cell hybrid specificities characterized by reactivity to either $\left[\mathrm{A}^{75}\right]$ GP68-84 or $\left[\mathrm{P}^{80}\right]$ GP68-84 (Fig. 2) were not detected in assays 

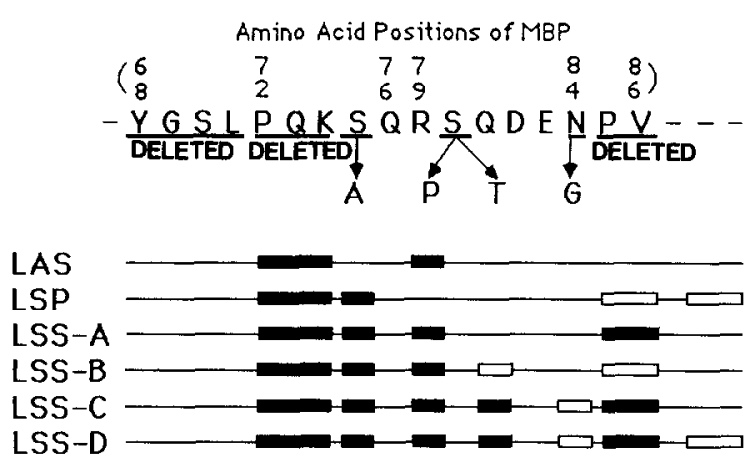

FIG. 6. Schematic representation of monoclonal determinants recognized by Lewis rat $\mathrm{T}$ cells. Seven deletions or substitutions of GP68-86 were studied to delineate monoclonal determinants of T hybrids. Each structural alteration was assessed by the comparative activities of the following peptides or MBPs: 68-71 deletion, GP68-86 versus GP72-86; 72-74 deletion, GP72-86 versus GP75-86; $\mathrm{A}^{75}$ substitution, GP68-84 versus $\left[A^{75}\right]$ GP68-84; $\mathrm{P}^{80}$ substitution, GP68-84 versus $\left[\mathrm{P}^{80}\right]$ GP68-84; $T^{80}$ substitution, GPMBP versus RMBP; $G^{84}$ substitution, GP68-86 versus $\left[G^{84}\right] G P 68-86$; and $85-86$ deletion, GP68-86 versus GP68-84. An additional structural requirement for residues $C$-terminal to $V^{86}$ was inferred by the comparative activities of GPMBP and GP68-86. Each of these structural modifications is diagrammatically represented in the sequence of GP68-86 at the top of the figure. Enclosed bars located directly below each structural modification represent instances in which the potency of the hybrid response (designated at the left of the figure) was reduced by over 100 -fold. Open bars represent structural changes that resulted in a 10- to 100-fold reduction in the potency of the hybrid response. Continuous lines indicate that a given structural change did not reduce the hybrid response by more than 10 -fold.

measuring the in vitro proliferation of LNC (Fig. 1). However, these specificities or very similar specificities were detected in previous studies focusing on the potentiation of EAE transfer activity by LNC (8). Third, consistent and significant differences in anti-RMBP reactivity were observed among different groups of hybrids (Figs. 25; see also summary in Fig. 7). The LAS, LSP, and LSS-A hybrids possessed relatively strong reactivity to RMBP whereas the LSS-B, $-C$, and -D hybrids possessed progressively weaker reactivity to RMBP. These differences were apparent whether evaluated relative to reactivity to GPMBP or in terms of the concentration of RMBP required for half-maximal responses (Figs. 2-5). Fourth, some determinants differed greatly in the number and layout of the structural modifications that impaired the respective hybrid response (Fig. 6). For example, the LAS determinant appeared to be discontinuous, since it required the $72-74$ sequence as well as a $S^{80} / T^{80}$ residue but was relatively unaffected by modifications affecting either position 75 or the 84-86 sequence. In contrast, the LSS-D determinant appeared continuous since it was disrupted by virtually every modification studied in this investigation.

The specific patterns of recognition by different hybrids in all likelihood reflected differences in clonally derived $\mathrm{T}$ cell receptor specificity. Other response parameters could not account for these specificity differences, since the hybrids generally did not differ either in their reactivity to GPMBP or in their ability to secrete IL 2 . The only exception was the LSS-B3 hybrid (Fig. 3) which was somewhat less reactive to GPMBP when compared to the other hybrids. Nevertheless, LSS-B3 had essentially the same specificity pattern as the other LSS-B hybrids, except that the pattern as a whole was right-shifted by approximately one order of magnitude. The observation that the hybrid responses could be readily classified into distinct specificity categories rein- 
forced the concept that $\mathrm{T}$ cell hybrids are a valid means to study the specificity of monoclonal $T$ cells. This has been verified in an investigation which showed that hybrids had essentially the same specificity as the $T$ cell clones from which they were derived (13).

The extent of clonal heterogeneity characterizing the anti-GP68-86 Lewis rat T cell response indicates that the susceptibility of Lewis rats to EAE is not due to a single aberrant clone which causes EAE after evading ontogenetic mechanisms of clonal deletion. Rather, this investigation indicates that multiple clones which express antiself RMBP reactivity have escaped clonal deletion and participate in an encephalitogenic immune response. Other investigations have also implicated clonal diversity in the $T$ cell response against encephalitogenic region of MBP in rats (12) and mice (14, 15). By use of highly purified synthetic peptides, this study has revealed a more diverse anti-GP68-86 T cell repertoire than previously anticipated while also providing detailed structural information on the antigenic determinants comprising the encephalitogenic region.

Since this investigation was focused upon $T$ cell hybrids, it was not feasible to directly assess the relation of a given clonotypic specificity with encephalitogenic activity. Nevertheless, the LAS and LSP hybrids (Fig. 2) exhibited specificities very similar to specificities previously shown to be expressed by LNC mediating potentiated EAE transfer activity (8). These specificities were characterized by potent reactivity to $\left[\mathrm{A}^{75}\right]$ GP68-84 and $\left[\mathrm{P}^{80}\right]$ GP68-84 peptides. Conversely, hybrids such as LSS-A and LSS-D exhibited specificities very similar to those expressed by LNC dominating the in vitro proliferative response to GP68-86 ((8) and Fig. 1). These LNC and hybrids did not exhibit detectable reactivity to either of the $\left[\mathrm{A}^{75}\right]$ - or $\left[\mathrm{P}^{80}\right] \mathrm{GP68-84}$ peptides. By these correlations, the LAS and LSP hybrids appear to represent the T cell subset mediating potentiated EAE transfer activity whereas the LSS-A and LSS-D hybrids represent the $\mathrm{T}$ cell subset dominating in vitro proliferation. The division of these respective hybrid specificity groups into discrete subsets has been confirmed by other criteria as well (manuscript in preparation).

To a large degree, this dissociation of EAE transfer and proliferative activity most likely reflects unique specificity patterns of functionally distinct $T$ cell subsets (8). However, other additional factors may contribute to the dissociation of EAE transfer and proliferative activities of LNC. For example, clonotypic differences in antiRMBP reactivity (Fig. 6) may be one of the factors explaining why EAE transfer activity is more closely correlated with the LAS and LSP specificities than with the LSS specificities. As illustrated in Fig. 7, the LAS and LSP hybrids exhibited potent anti-RMBP reactivity whereas the LSS hybrids, with the exception of LSS-A, exhibited less potent anti-RMBP reactivity. If one assumes that the potency of the antiRMBP response in vitro is directly related to the clone's ability to recognize RMBP in vivo, then recognition of RMBP in vivo would in turn constitute one of the essential factors controlling the respective clone's ability to mediate EAE. Even though the LAS and LSP represented only 3 of the 12 hybrids, these T cells nevertheless dominated the hybrid response to low concentrations of RMBP. Since recognition of RMBP in the CNS target tissue is a prerequisite for EAE-inducing activity, the normal counterparts of these hybrids may possess exceptionally high levels of encephalitogenic activity. Conversely, the LSS-B, $-\mathrm{C}$, and -D hybrids were only marginally reactive with RMBP (Figs. 3-5), suggesting their normal counterparts, on a per cell basis, may possess relatively low levels of encephalitogenic activity. 


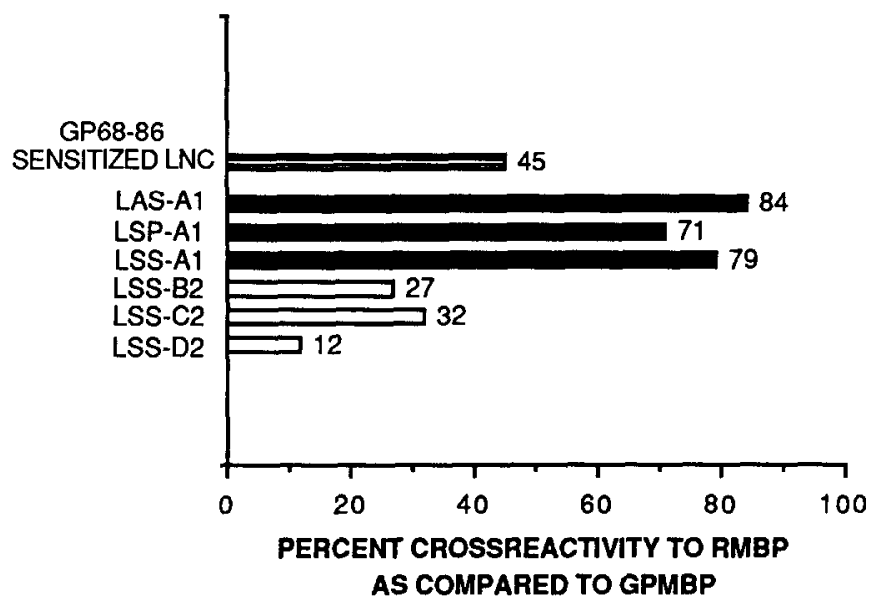

FIG. 7. Differences in hybrid reactivity to RMBP. For GP68-86-sensitized LNC and each of the representative hybrids, the responses at $0.1,1,10$, and $100 \mathrm{nM}, 1$, and $10 \mu \mathrm{M}$ of RMBP are summed (minus background) and then divided by the summed response to the same concentrations of GPMBP. Interestingly, the anti-RMBP response of LNC appears intermediate to the two groups of hybrids exhibiting low and high levels of reactivity to RMBP.

The similarity of the specificities expressed by LSP hybrids and encephalitogenic LNC can be readily understood, since these hybrids apparently did not recognize the residue at position 80 . These hybrids thereby reacted strongly with antigens such as RMBP, $\left[\mathrm{P}^{80}\right] \mathrm{GP} 68-84$, and GP68-84 that differed only in the residue at position 80 . Less obvious is the association of LAS and LSS-A specificities with potent anti-RMBP reactivity, since these hybrids did not respond to $\left[\mathrm{P}^{80}\right]$ GP68-84 to any extent. These hybrids were apparently more permissive of the conservative $T^{80}$ substitution than the divergent $P^{80}$ substitution.

The observed clonotypic differences in anti-RMBP reactivity may also account, in part, for why GPMBP is more encephalitogenic than RMBP in rats $(1,16)$. Sensitization with GPMBP would result in the in vivo recruitment of highly reactive antiRMBP clones in addition to clones having only marginal reactivity against RMBP, since most of the hybrids cxhibited potent reactivity to GPMBP (Figs. 2-5). The encephalitogenic potential of these marginally reactive anti-RMBP clones may be substantial, since their inherently low autoreactivity may be offset by their numerical predominance. Sensitization with RMBP would be a far less effective stimulus for the activation and in vivo recruitment of low-affinity anti-RMBP/Ia $\mathrm{T}$ cell clones. Hence, the clonotypes represented by the LSS-B, -C, and -D hybrids (Figs. 3, 4, and 5 , respectively) would contribute far more to an encephalitogenic immune response induced by GPMBP than one induced by RMBP.

Different hybrid specificities were also distinguished by deletions of the C-terminal 85-86 sequence and $\mathrm{N}^{84}$ side chain. In fact, both LSP and LSS-D hybrids appeared to require residues beyond the $\mathrm{C}$-terminal $\mathrm{V}^{86}$ for full activity (Fig. 6). On the other hand, the LAS-Al hybrid was exceptional in that it did not discriminate between GP68-84, [G $\left.{ }^{84}\right]$ GP68-86, and GP68-86. These findings confirm previous indications that at least two populations of $T$ cells could be defined by differential requirements for C- and N-terminal residues of GP72-86 (3). The contrast between the LAS and 
LSS-D determinants is intriguing since the former had a concise, centrally located format whereas the latter had a broadly dispersed format. These contrasting views of determinant structure may reflect a bias in the set of synthetic peptides used to define the determinants, or alternatively, a qualitative difference in the respective determinants. For example, LAS may recognize an $\alpha$-helical determinant whereas LSS-D may recognize a determinant having essentially a native conformation.

Overall, this investigation has documented the oligoclonal heterogeneity of the anti-GP68-86 repertoire of Lewis rat T cells while also providing a more precise delineation of the multiple, overlapping determinants in the encephalitogenic region of MBP. Furthermore, this study has shown that the specificity of the LAS and LSP T cell hybrids represents the unique LNC specificity previously associated with the potentiated transfer of $\mathrm{EAE}(8)$.

\section{ACKNOWLEDGMENTS}

The authors express deep appreciation for the expert technical assistance provided by Mrs. Maxine Solvay and Ms. Louise Pope. The authors thank Dr. Roderick Nairn for his careful critiquing of this manuscript.

\section{REFERENCES}

1. Kibler, R. F., Fritz, R. B., Chou, F. C.-H., Chou, C.-H. J., Peacocke, N. Y., Brown, N. M., and McFarlin, D. E., J. Exp. Med. 146, 1323, 1977.

2. Chou, C.-H. J., Chou, F. C.-H., Kowalski, T. J., Shapira, R., and Kibler, R. F., J. Neurochem. 28, 115 , 1977.

3. Chou, C.-H. J., Fritz, R. B., Chou, F. C.-H., and Kibler, R. F., J. Immunol. 123, 1540, 1979.

4. Ben-Nun, A., and Cohen, I. R., J. Immunol. 128, 1450, 1982.

5. Vandenbark, A. A., Offner, H., Reshef, T., Fritz, R., Chou, C.-H. J., and Cohen, I. R., J. Immunol. 135, 229, 1985.

6. Mannie, M. D., Paterson, P. Y., U'Prichard, D. C., and Flouret, G., Proc. Natl. Acad. Sci. USA 82, $5515,1985$.

7. Kies, M. W., Springer Semin. Immunopathol. 8, 295, 1985.

8. Mannie, M. D., Paterson, P. Y., U'Prichard, D. C., and Flouret, G., J. Immunol. 142, 2608, 1989.

9. Swanborg, R. H., Swierkosz, J. E., and Saieg, R. G., J. Immunol. 112, 594, 1974.

10. Eylar, E. H., Brostoff, S., Hashim, G., Caccam, J., and Burnett, P., J. Biol. Chem. 246, 5770, 1971.

11. Prud'homme, G. J., Fuks, A., Guttmann, R. D., and Colle, E., J. Immunol. 136, 1535, 1986.

12. Happ, M. P., and Heber-Katz, E., J. Exp. Med, 167, 502, 1988.

13. Ozaki, S., Durum, S. K., Muegge, K., York-Jolley, J., and Berzofsky, J. A., J. Immunol. 141, 71, 1988.

14. Huang, S. K., and Sriram, S., Immunogenetics 27, 370, 1988.

15. Zamvil, S. S., Mitchell, D. J., Lee, N. E., Moore, A. C., Waldor, M. K., Sakai, K., Rothbard, J. B., McDevitt, H. O., Steinman, L., and Acha-Orbea, H., J. Exp. Med. 167, 1586, 1988.

16. McFarlin, D. E., Blank, S. E., Kibler, R. F., McKneally, S., and Shapira, R., Science 179, 478, 1973. 